\title{
MOA-2011-BLG-028Lb: A NEPTUNE-MASS MICROLENSING PLANET IN THE GALACTIC BULGE*
}

\author{
J. Skowron ${ }^{1}$, A. Udalski ${ }^{1}$, R. Poleski ${ }^{1,2}$, S. KozŁowski ${ }^{1}$, M. K. Szymański ${ }^{1}$, Ł. Wyrzykowski ${ }^{1}$, K. Ulaczyk ${ }^{3}$, \\ P. Pietrukowicz ${ }^{1}$, G. PietrZYŃSKi ${ }^{1}$, I. SosZYŃSKI ${ }^{1}$ \\ (The OGLE Collaboration) $)^{20}$, \\ F. Abe ${ }^{4}$, D. P. Bennett ${ }^{5}$, A. Bhattacharya ${ }^{5}$, I. A. Bond ${ }^{6}$, M. Freeman ${ }^{7}$, A. Fukui ${ }^{8}$, Y. Hirao ${ }^{9}$, Y. Itow ${ }^{4}$, N. Koshimoto ${ }^{9}$, \\ C. H. Ling $^{6}$, K. Masuda ${ }^{4}$, Y. Matsubara ${ }^{4}$, Y. Muraki ${ }^{4}$, M. Nagakane ${ }^{9}$, K. Ohnishi ${ }^{10}$, N. Rattenbury ${ }^{7}$, To. Saito ${ }^{11}$, \\ D. J. Sullivan ${ }^{12}$, T. Sumi ${ }^{9}$, D. Suzuki ${ }^{5}$, P. J. Tristram ${ }^{13}$, A. Yonehara ${ }^{14}$ \\ (The MOA Collaboration) ${ }^{21}$ \\ AND

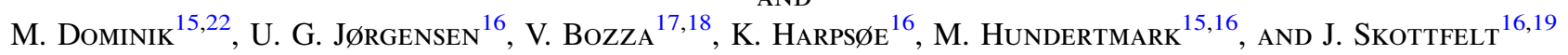 \\ (The MiNDSTEp COLlaboration) \\ ${ }^{1}$ Warsaw University Observatory, Al. Ujazdowskie 4, 00-478 Warszawa, Poland; jskowron@astrouw.edu.pl \\ ${ }^{2}$ Department of Astronomy, Ohio State University, 140 W. 18th Avenve, Columbus, OH 43210, USA \\ Department of Physics, University of Warwick, Gibbet Hill Road, Coventry, CV4 7AL, UK \\ ${ }^{4}$ Solar-Terrestrial Environment Laboratory, Nagoya University, Nagoya 464-8601, Japan \\ ${ }^{5}$ Department of Physics, University of Notre Dame, Notre Dame, IN 46556, USA \\ ${ }^{6}$ Institute of Information and Mathematical Sciences, Massey University, Private Bag 102-904, North Shore Mail Centre, Auckland, New Zealand \\ 7 Department of Physics, University of Auckland, Private Bag 92019, Auckland, New Zealand

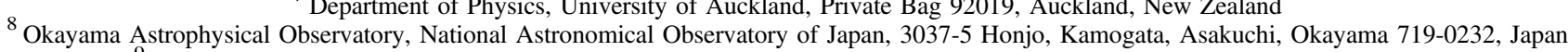 \\ ${ }^{9}$ Department of Earth and Space Science, Graduate School of Science, Osaka University, Toyonaka, Osaka 560-0043, Japan \\ ${ }^{110}$ Nagano National College of Technology, Nagano 381-8550, Japan \\ ${ }^{11}$ Tokyo Metropolitan College of Aeronautics, Tokyo 116-8523, Japan \\ ${ }^{12}$ School of Chemical and Physical Sciences, Victoria University, Wellington, New Zealand \\ ${ }^{13}$ Mt. John University Observatory, P.O. Box 56, Lake Tekapo 8770, New Zealand \\ ${ }^{14}$ Department of Physics, Faculty of Science, Kyoto Sangyo University, 603-8555 Kyoto, Japan \\ ${ }^{15}$ SUPA, University of St Andrews, School of Physics \& Astronomy, North Haugh, St Andrews, KY16 9SS, UK \\ ${ }^{16}$ Niels Bohr Institute \& Centre for Star and Planet Formation, University of Copenhagen, Østervoldgade 5, DK-1350 Copenhagen K, Denmark \\ ${ }^{17}$ Dipartimento di Fisica "E.R. Caianiello," Università di Salerno, Via Giovanni Paolo II 132, I-84084, Fisciano (SA), Italy \\ ${ }^{18}$ Istituto Nazionale di Fisica Nucleare, Sezione di Napoli, I-80126 Napoli, Italy \\ ${ }^{19}$ Centre for Electronic Imaging, Dept. of Physical Sciences, The Open University, Milton Keynes MK7 6AA, UK \\ Received 2015 December 16; accepted 2016 February 3; published 2016 March 10
}

\begin{abstract}
We present the discovery of a Neptune-mass planet orbiting a $0.8 \pm 0.3 M_{\odot}$ star in the Galactic bulge. The planet manifested itself during the microlensing event MOA-2011-BLG-028/OGLE-2011-BLG-0203 as a low-mass companion to the lens star. The analysis of the light curve provides the measurement of the mass ratio $(1.2 \pm 0.2) \times 10^{-4}$, which indicates that the mass of the planet is 12-60 Earth masses. The lensing system is located at $7.3 \pm 0.7 \mathrm{kpc}$ away from the Earth near the direction of Baade's Window. The projected separation of the planet at the time of the microlensing event was 3.1-5.2 au. Although the microlens parallax effect is not detected in the light curve of this event, preventing the actual mass measurement, the uncertainties of mass and distance estimation are narrowed by the measurement of the source star proper motion on the OGLE-III images spanning eight years, and by the low amount of blended light seen, proving that the host star cannot be too bright and massive. We also discuss the inclusion of undetected parallax and orbital motion effects into the models and their influence onto the final physical parameters estimates.
\end{abstract}

Key words: gravitational lensing: micro - planetary systems

\section{INTRODUCTION}

Both the extrasolar planet distribution and the planet formation mechanisms are of a great interest in current astrophysics. To gain insight into these matters all possible methods of planet detection should be exercised, since every method has its own strengths and biases, and probes a particular subspace of the planetary system parameters.

\footnotetext{
* Based on observations obtained with the $1.3 \mathrm{~m}$ Warsaw telescope at the Las Campanas Observatory operated by the Carnegie Institution of Washington.

${ }^{20}$ Optical Gravitational Lensing Experiment (OGLE).

21 Microlensing Observations in Astrophysics (MOA) Collaboration.

22 Royal Society University Research Fellow.
}

The core-accretion theory of planet formation (Laughlin et al. 2004; Ida \& Lin 2005) predicts that giant planets and Neptune-mass planets form beyond the snow line of their hosts where the solid material density is greatly increased by frosting. Although transit and radial velocity (RV) methods of planet detection find a number of giant extrasolar planets, these are mainly hot Jupiters that migrated from the place of their formation. Sensitivity of the aforementioned methods to planets with orbits of a few au and larger, is very limited; typical sensitivity ends below 2.5 au (Johnson et al. 2010). For example, Cumming et al. (2008) studied periods of 2-2000 days with the $\mathrm{RV}$ method, which corresponds to the mean semimajor axis of $0.31 \mathrm{au}$, while the position of the snow line can be approximated with $\sim 2.7 M / M_{\odot}$ (Kennedy \& Kenyon 2008). 
The microlensing method is best suited for probing the planet population beyond the snow line, being sensitive to gas giant planets as well as Neptune-mass planets in the region of their formation (see Gaudi 2012, for a review). In the Galacticscale lensing event, the light from the distant star, bent by the gravity of a stellar-mass lens, typically passes $2-4$ au from this lens. If a planetary-mass companion to the lens is present at these separations, it can disturb the image of the distant star, change its magnification, and therefore manifest its own presence to the careful observer.

The lensing action does not depend on the light of the host star, the planet, or their radii, and is only a weak function of their mass $(\propto \sqrt{M})$. This gives the microlensing method a great advantage in discovering cold planets around all types of stars.

To date, more than $40 \%$ of planets found by microlensing can be classified as cold Neptunes or sub-Saturns and $\sim 30 \%$ as giant planets. Initial studies of the sample of microlensing planets show that $38_{-22}^{+31} \%$ of stars host cold super-Earths or Neptunes with separations in the 1.6-4.3 au range (Gould et al. 2006) and that they are $7_{-3}^{+6}$ times more common than cold Jupiters (Sumi et al. 2010).

Although the Kepler mission provided evidence that the Neptune-mass planets are common on shorter-period orbits, the gravitational lensing results strongly suggest that this is also the case for longer-period orbits. Furthermore, studies of the planetary mass function based on the microlensing sample (Gould et al. 2010; Cassan et al. 2012) confirm the increased abundance of planetary companions beyond the snow line (see Figure 8 of Gaudi 2012) where they are expected to form efficiently.

Despite only about 30 planets being known from the microlensing technique to date, it has already proved itself as a complementary to other methods of planet discovery and has provided useful insights into the planetary population in the Galaxy (Gaudi 2012). It is crucial, however, to do further work on expanding the sample of microlensing planet to facilitate these studies. On one hand, the statistical strength of the arguments should be greatly improved; on the other hand, the interesting edge cases are being found in the process: planets in binary stars systems (e.g., Gould et al. 2014; Poleski et al. 2014a; Udalski et al. 2015a) or giant planets around low-mass stars (e.g., Koshimoto et al. 2014; Poleski et al. 2014b; Fukui et al. 2015; Skowron et al. 2015). Together with the sound statistics for more typical planetary systems, it will be possible to predict the underlying frequency of these unusual systems.

Here we report the discovery of the $\sim 30 M_{\oplus}$ planet (MOA2011-BLG-028Lb), which is most likely located in the Galactic bulge and orbits the moderately massive star $\left(\sim 0.75 M_{\odot}\right)$ at $\sim 3-5 \mathrm{au}$. Because of the distant position of the planetary system in the Galaxy (the small parallax) and low-magnification nature of the event (the high uncertainties in the flux estimations), its physical parameters could not be accurately derived from the light curve. Nevertheless, we provide the best estimations based on our understanding of the Galaxy, the measured source star proper motion (from 8 years of the OGLE monitoring), and the upper limits on the lens flux from the OGLE light curve. We hope that future high-resolution imaging can provide additional constraints.

In Section 2 we describe the photometric observation and the light curves from each data set taken into account. Section 3 presents the microlensing model parametrization and discusses additional effects taken into account when modeling the light curve data. The physical parameters of the system are derived in Section 4 through the use of some additional input and assumptions about the Galaxy and the source star. Results are presented in Section 5 together with the discussion of future follow-up observations. Conclusions are provided in Section 6.

\section{PHOTOMETRIC OBSERVATIONS AND LIGHT- CURVE DATA}

In 2011 March the Microlensing Observations in Astrophysics group (MOA) announced the candidate microlensing event MOA-2011-BLG-028 ${ }^{23}$ on their Microlensing Alerts webpage. $^{24}$ This event was also monitored by the fourth phase of the Optical Gravitational Lensing Experiment (OGLE-IV, Udalski et al. 2015b) and subsequently announced by the Early Warning System (EWS) ${ }^{25}$ as OGLE-2011-BLG-0203 ${ }^{26}$ in the batch of the 431 microlensing events initializing the EWS at the OGLE-IV phase.

The Event's coordinates are $(\alpha, \delta)_{\mathrm{J} 2000}=\left(18^{\mathrm{h}} 03^{\mathrm{m}} 24^{\mathrm{s}} .96\right.$, $\left.-29^{\circ} 12^{\prime} 48^{\prime \prime} 3\right)$ in equatorial coordinates and $(l, b)=$ $(1.7,-3.5)$ in Galactic coordinates. (The accuracy of the absolute position is of the order of 0.1 arcsec.)

The object was magnified from 2010 December until 2011 September and the magnification peaked on 2011 April 22 $\left(\mathrm{HJD}^{\prime}=\mathrm{HJD}-2450000=5674\right) \quad 0.4 \mathrm{mag}$ brighter than the baseline level of $I=15.3$. Twenty days after the peak, on May $12-14\left(\mathrm{HJD}^{\prime}=5694.2-5696.7\right)$, the short-time planetary anomaly was recorded by OGLE, MOA, and Danish telescopes. The anomaly was spotted a couple of days after it was already finished and the event followed a typical Paczyński light curve (Paczyński 1986) after that. No other observatories managed to gather additional data on the planetary signal in the event's light curve.

Figure 1 shows the 1.5 year-long section of the light curve covering the microlensing event and the planetary anomaly. This object was monitored by OGLE from 2001 and by MOA from 2006 and does not show any other variability outside of the period shown.

The OGLE survey uses the dedicated $1.3 \mathrm{~m}$ Warsaw Telescope located at Las Campanas Observatory in Chile. The MOA group observes with the $1.8 \mathrm{~m}$ telescope at Mt. John University Observatory in New Zealand. The $1.5 \mathrm{~m}$ Danish telescope at ESO La Silla in Chile is operated by the MiNDSTEp Consortium. ${ }^{27}$

While the microlensing survey groups (OGLE and MOA) constantly monitor the relevant regions of the Milky Way to identify and characterize microlensing events, the MiNDSTEp Consortium and other follow-up teams monitor only promising microlensing events in an effort to detect extrasolar planets (Dominik et al. 2010). It happened that the automatic prioritization algorithm used by this group fortunately flagged the MOA-2011-BLG-028 as a potentially interesting event to observe right before the actual planetary anomaly occurred. The first data point from the Danish telescope considered in this work is actually already during the planetary anomaly (at $\left.\mathrm{HJD}^{\prime}=5694.84\right)$.

\footnotetext{
23 https://it019909.massey.ac.nz/moa/alert/display.php?id=gb13-R-3-8819

24 https://it019909.massey.ac.nz/moa/alert/alert2011.html

25 http://ogle.astrouw.edu.pl/ogle4/ews/ews.html

26 http://ogle.astrouw.edu.pl/ogle4/ews/2011/blg-0203.html

27 http://www.mindstep-science.org/about_us.2011.html
} 

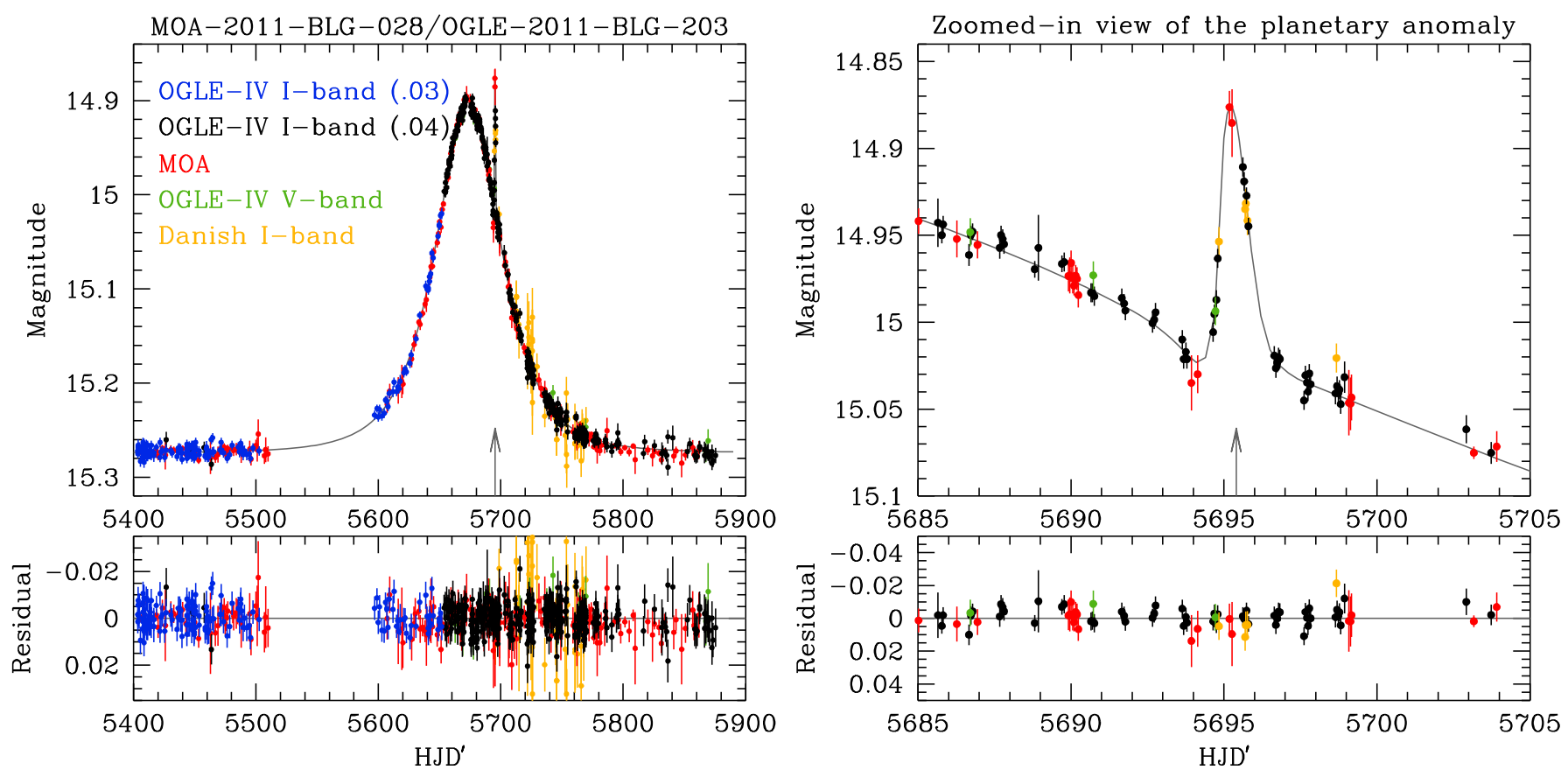

Figure 1. Left panel: 1.5 year-long section of the light curve of the MOA-2011-BLG-028/OGLE-2011-BLG-0203. Right panel: 20 day section centered around the planetary anomaly (marked by an upward arrow). The whole OGLE light curve for this object spans 15 years. We only show the data that were used in the final modeling. The black line marks the best-fit microlensing model where the light of a Galactic bulge giant is magnified for $\sim 200$ days (around 2011 April $22 \mathrm{nd}$ ) by a stellar object near the light's path and is additionally disturbed for $\sim 2$ days (around 2011 May 13th) by a low-mass companion of that object. Five data sets are represented as color dots together with error bars used in the fitting process. The magnitude scale is calibrated to the OGLE-III photometric map. The light curve of the second solution $\left(u_{0}<0\right)$ is very similar and indistinguishable by eye on this plot; hence, only one solution $\left(u_{0}>0\right)$ is presented. The bottom part of each plot shows residuals against the best-fit model. (HJD ${ }^{\prime}=$ HJD-2450000). OGLE-IV I-band light curve is split into the measurements made with the CCD detectors no. 03 and 04 of the OGLE-IV camera. Up to $\mathrm{HJD}^{\prime}=5650$ the majority of the measurements are done with the detector no. 03, while after that date, due to the slight change in the pointing model of the telescope, all measurements, including the peak of the event and planetary anomaly, have fallen onto detector no. 04 .

In this work, we also use data from the previous phase of the OGLE survey (OGLE-III) that was operating from 2001 to 2009 at the $1.3 \mathrm{~m}$ Warsaw telescope. The $V$ - and $I$-band data comes from the project's final data reductions (Udalski et al. 2008). The calibrated data on the stars in the neighborhood of the event are taken from the Galactic bulge photometric maps (Szymański et al. 2011). Thus, all OGLE magnitudes reported in this paper are standard $V$ (Johnson) and $I$ (Cousins) magnitudes.

\subsection{Data Preparation}

In the light curves from both microlensing surveys, the main event is clearly detected and shows all prerequisites of the microlensing event by a stellar system. The planetary anomaly is detected by three telescopes and its shape is what we expect from the added magnification of the major image by the planetary companion (e.g., Mao \& Paczyński 1991).

We expect that the projected position of the planetary system on the plane of the sky can be modified during the curse of the event ( 200 days) by its orbital motion. Also, the motion of the observer on Earth's orbit can modify the geometry of the event via the so-called microlens parallax effect.

The decade-long light curve of the stars involved in the event show no signs of periodic or non-periodic variability nor any transient outbursts. Since we expect the microlensing to be the source of the detected magnification, we fit the microlensing model to all data sets and require all data sets to yield $\chi^{2}$ per degree of freedom equal to unity, which is done by rescaling the uncertainties. We use the standard approach to measurement errors rescaling as described by Skowron et al. (2011, Section 2.2).

Theoretically founded expectations about the shape of the light curve in conjunction with the great redundancy of the data allows us to easily judge which data points can be outright classified as outliers. Also, it is possible to identify suspect spans of the light curve data taken under sub-optimal conditions for further evaluation and possible removal from the final data set. Because we are seeking slow-evolving trends in the light curve characteristic for the parallax effect or lens system orbital motion, aside from the short-term planetary anomaly, we are conservative and remove all suspect data.

\subsection{OGLE-IV Light-curve Data}

The object described in this paper falls into the gap between two CCD detectors of the OGLE-IV camera in the standard field no. BLG512 of the Galactic bulge survey. It is also located close to the corners of both detectors. See Figure 2 for a detailed view. Fortunately, the typical pointing scatter of the telescope (rms $\sim 60 p x$ ) causes some number of measurements to be done with the detector no. 03 and some with the detector no. 04. The original OGLE-IV BLG512 pointing in 2010 caused the star to fall more often in the 03 detector. However, after early 2011 adjustments of the position of the OGLE-IV high cadence fields, including BLG512, the majority of the observations of the star were recorded on the 04 detector. Finally, a small temporary adjustment to the BLG512 field (about 50 pixels) was additionally introduced after the discovery of the planetary anomaly to secure good coverage 


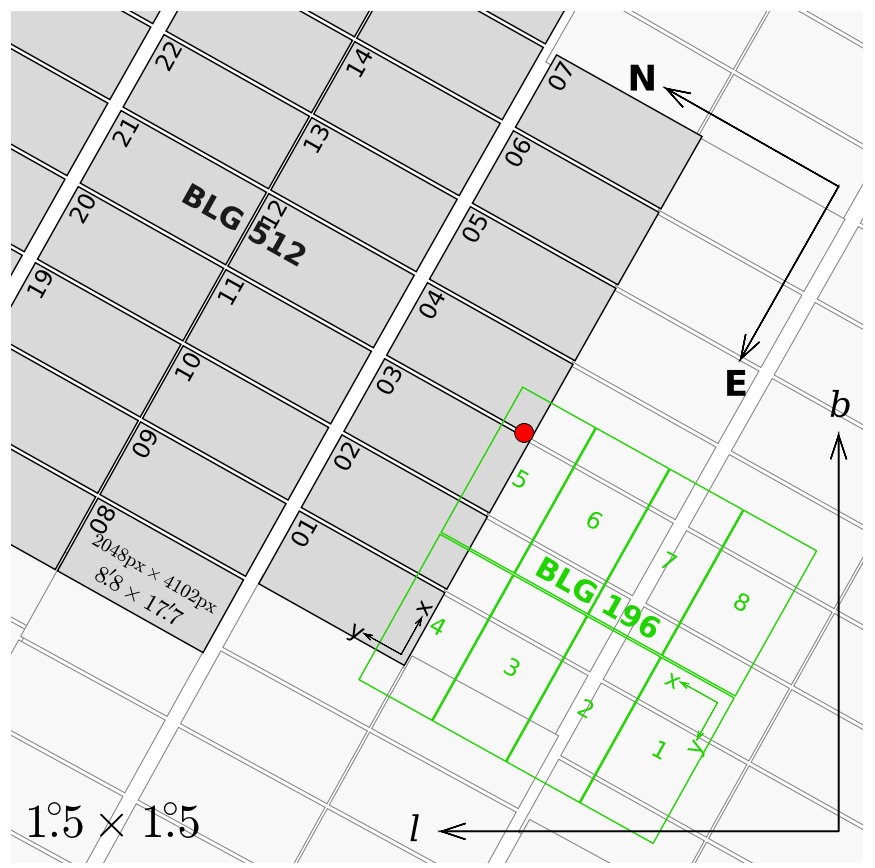

Figure 2. Location of the microlensing event MOA-2011-BLG-028/OGLE2011-BLG-0203 in respect to the OGLE-IV field BLG512 and the OGLE-III field $B L G 196$. The red circle has a radius of 1 arcmin and marks the position of the event. In the OGLE-IV survey, the event falls into the gap between the CCD detectors number 03 and 04 , close to the corners of both detectors. Because of the scatter in the telescope pointing, the microlensing event was registered on both CCD detectors. It was also automatically discovered by the early warning system. The measurements, however, are split into two separate light curves, for both detectors, and require additional cross-calibration. Earlier, during the course of the OGLE-III survey, the region of the future microlensing event was monitored for 8 years with the CCD detector number 5 in the field BLG196. Galactic north is up and Galactic east is to the left. The plot has a $1.5 \times 1.5$ field of view. Each CCD detector covers $8.8 \times 17 ! .7$ of the sky.

of the late stages of the microlensing event. Since this field is monitored with a high cadence of up to 10-30 exposures per night, the two data sets combined yield good continuous coverage of the event throughout the season, albeit with a lower cadence of $\sim 2$ per night.

The routine OGLE-IV calibrations to the Johnson-Cousins photometric system do not perform well for this object due to its extreme position (at the edge and near the corner of the detector). Fortunately, this region of the sky was also densely monitored by the previous phase of the OGLE survey (OGLEIII, cf. Figure 2). Where the object in question was wellmeasured with over 1300 individual observation during eight years of the project and its calibrated $I$ - and $V$-band magnitudes are given by Szymański et al. (2011): $(V-I, I)=(1.829,15.275) \pm(0.011,0.006)_{\text {stat }}$.

The magnified source star is most likely a red clump giant in the Galactic bulge (see Figure 3) and accounts for the bulk of light seen before and after the event (the microlensing models with very low amounts of unmagnified light are strongly preferred by the light curve; see Section 3). Hence, we can calibrate the whole OGLE-IV light curve to the OGLE-III Johnson-Cousins magnitudes, as measured before the event, and not worry about the color changes during the event and thus, the different color terms. Any potential errors this procedure introduces are insignificant compared with the OGLE-III calibration uncertainties (0.01-0.02 mag).

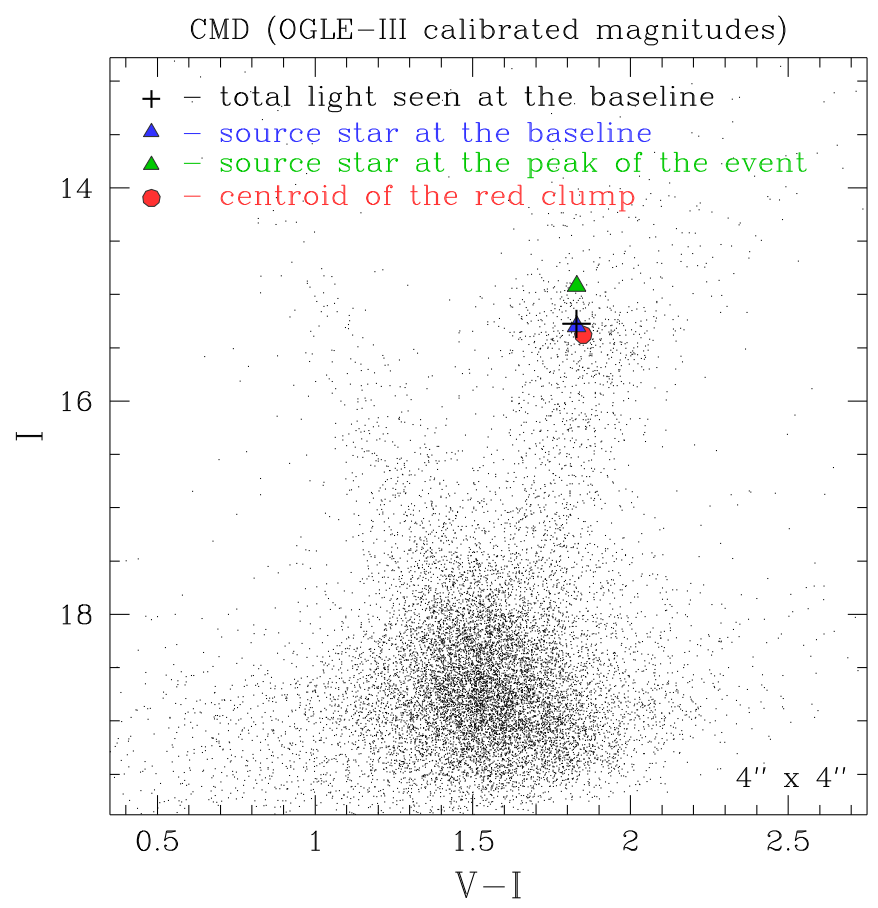

Figure 3. Color-magnitude diagram (CMD) based on the OGLE-III photometric map (Szymański et al. 2011) of the $4^{\prime} \times 4^{\prime}$ region around the source star. The center of the red clump giant's region is marked with a red circle. The position of the studied object (at the baseline) is marked with a blue triangle. The microlensing model strongly favors solutions with small amounts of additional light (blended light, see Table 1). Therefore, the light we see at the baseline is mainly the light of the microlensed star. We infer from its position on the CMD that it is most likely a red clump giant in the Galactic bulge.

To accurately measure the I-band brightness evolution during the event, we construct a custom template image by averaging a dozen science frames taken under good weather conditions and where the object position fallen not less then 0.5 arcmin from the edge of the detector. The centroid of the source star is calculated from seven frames in 2011 April when the magnification was the highest. Then we perform an optimized photometry with the OGLE-IV pipeline (based on the Difference Image Analysis (DIA) Woźniak 2000) measuring the variable light of the event with profile photometry on the subtracted image at the fixed position of the source. The resulting OGLE-IV light curve consists of 1974 measurements with CCD detector no. 04 and 275 measurements with detector no. 03, out of which we remove one data point and 25 data points, respectively, as outliers or data taken under sub-optimal conditions. We rescale the error bars by adding in quadrature 3 mmag and $3.5 \mathrm{mmag}$, respectively.

The $V$-band observation are reduced with the standard image subtraction pipelines, as described by Udalski (2003), from the measurements made with the CCD detector no. 04. There are 63 measurements after one outlier is removed, and the error bars are adjusted by adding 6 mmag scatter in quadrature.

\subsection{MOA Light-curve Data}

The MOA data were reduced using the standard image subtraction pipeline used by the survey group and described by Bond et al. (2001). We fit for possible airmass and seeing correlations correct the standard photometry accordingly.

The MOA light curve consists of 7183 measurements, from which we take only 5904 with the reported uncertainties lower 
than $0.01 \mathrm{mag}$. The typical sampling rate during the observing season was $\sim 5$ per night. Due to the season-to-season lowamplitude shifts $(\sim 0.01 \mathrm{mag})$ present in the light curve-most likely an instrument-introduced systematic effect-we remove all data outside of the 2011 season to avoid spurious signals in the microlens parallax measurement. We measured that different baseline levels between 2010 and 2011 seasons were introducing asymmetricity to the light curve that resulted in the apparent $2-\sigma$ detection of the parallax signal. This signal vanished when the MOA baseline data were cropped.

We also skip observations at the very beginning and at the end of the season, since these are not crucial for characterizing the event (due to the existence of other data), but carry some risk of introducing additional systematic errors. The MOA telescope on average observes worse weather than the Chilean sites and the high airmass and high effective seeing near the seasonal break, in conjunction with the large pixel size and significant crowding toward this pointing, makes the measurements more challenging.

From the HJD' range of 5620-5845, we take 784 data points and bin them in one-day intervals. We leave the period \pm 5 days around the planetary anomaly not binned, since the variations of the light curve in this region have a shorter timescale. Such a constructed light curve consists of 131 data points and the error bar scaling factor used is 2.0 .

\subsection{5 m Danish Telescope Light-curve Data}

The follow-up monitoring of the MOA-2011-BLG-028 event by the Danish telescope at the ESO Observatory, La Silla, Chile started before the planetary anomaly happened and was the result of the strategy described by Dominik et al. (2010) in which a large fraction of promising microlensing events discovered by the survey groups are monitored with moderate cadence in the anticipation that one of them will unveil the existence of a planetary companion. This serendipitously happened for this event.

Unfortunately, the event took place at the same time as the initial tests of the newly installed lucky imaging (LI) camera. Therefore, half of the observations were done with the old conventional CCD camera and half with the new EMCCD (LI) camera. Recent observations have demonstrated the ability to obtain photometry as accurate with the LI technique as with conventional CCDs at the same time as benefiting from the high speed and increased spatial resolution of the LI technique (Harpsøe et al. 2012; Skottfelt et al. 2015); the LI camera is now the standard instrument during the MiNDSTEp microlensing observations. The LI light curve started before the anomaly while first data from the standard CCD camera are at the rising part of the anomaly (as shown on the second panel of Figure 1). The mixed approach lowered the cadence of each data set and because of the difficulties in cross-calibrating both techniques from this early testing phase, we must treat the two light curves as separate data sets. The LI light curve shows significantly larger scatter then the standard one, and we do not use it in the modeling.

Albeit lower cadence, the standard I-band light curve is still useful to further confirm the amplitude and timing of the anomaly, as the Danish telescope is the third telescope to have observed it. Out of 57 data points, we remove seven as clear outliers (four solitary points and three consecutive points during a single bad night, unsupported by the data from other telescopes) and increase the error bars of the remaining 50 data points by a factor of 2.55 to ensure $\chi^{2}$ per degree of freedom $\sim 1$.

\section{THE LIGHT-CURVE MODELING}

\subsection{Microlensing Equations and Parameters}

A microlensing event is a transient magnification of light coming from the distance star (source of light: $\mathrm{S}$ ) by a massive object passing near the line of sight (the lens: L). Magnification is a direct result of stretching, bending, and increasing the number of source star's images on the sky by gravitational influence of the lens object.

In the case of perfect alignment of the observer, the lens, and the source, the observer sees one image in the shape of a ring around the lens - called the Einstein ring-and its angular radius on the sky $\left(\theta_{\mathrm{E}}\right)$ depends on the distances $\left(D_{\mathrm{L}}\right.$ and $\left.D_{\mathrm{S}}\right)$ and the mass of the lens $\left(M_{\mathrm{L}}\right)$ in the following way.

$$
\theta_{\mathrm{E}}=\sqrt{\kappa M_{\mathrm{L}}\left(\mathrm{AU} / D_{\mathrm{L}}-\mathrm{AU} / D_{\mathrm{S}}\right)}
$$

where $\kappa=8.144$ mas $M_{\odot}{ }^{-1}$ (cf. Gould 2000). Toward the Galactic bulge, most of the potential source stars are located at $D_{S} \sim 8 \mathrm{kpc}$ and lens stars are typically at $D_{L} \sim 4-7 \mathrm{kpc}$, hence, for stellar-mass lenses $\theta_{\mathrm{E}} \sim 0.5-1.0$ mas. Typically the relative lens-source proper motion in the Galaxy is of the order of $\mu_{\text {rel }} \sim 2-5$ mas $\mathrm{yr}^{-1}$ (for disk-bulge lensing events it is $\sim 4$ mas yr $^{-1}$ and for bulge-bulge lensing events it is

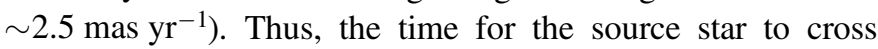
the Einstein ring of the lens is

$$
t_{\mathrm{E}}=\frac{\theta_{\mathrm{E}}}{\mu_{\mathrm{rel}}} \sim 20-100 \text { days },
$$

and is called the Einstein ring crossing time or the "Einstein time." The magnification during the microlensing events is, therefore, evolving on the timescale of days and months. It reaches its maximal value at the time, $t_{0}$, when the projected distance between the lens and the source star becomes minimal: denoted with $u_{0}$ and expressed in the units of $\theta_{\mathrm{E}}$. By convention we reserve the positive (negative) values for the $u_{0}$ parameter for cases in which the lens is passing the source on its right (left). See Figure 4 for reference.

It is sometimes beneficial to introduce the value of $t_{\mathrm{eff}}=u_{0} t_{\mathrm{E}}$ to be used as a model parameter in the fitting process instead of $t_{\mathrm{E}}$ or $u_{0}$.

The three introduced parameters $\left(t_{\mathrm{E}}, t_{0}\right.$, and $\left.u_{0}\right)$ or equivalently ( $t_{\mathrm{E}}, t_{0}$, and $\left.t_{\mathrm{eff}}\right)$, describe the time evolution of the magnification during the simplest microlensing event where both lens and source are single stars and the projected relative motion can be approximated as rectilinear (Paczyński 1986).

The existence of the planetary companion to the lens can influence the magnification pattern in its vicinity and therefore require an introduction of additional parameters to describe the observed light curve well. These paramenters are as follows: $s_{0}$ - the projected separation of the host star and its companion at some specified time ( $t_{0, \text { orb }}$, here fixed to 2455678.0 ); $q-\mathrm{a}$ mass ratio of the planet to its host; and $\alpha_{0}$-an angle of the skyprojected planet-host axis at the specified time $t_{0, \text { orb }}$, measured counter-clockwise from the direction of the lens-source relative proper motion $\boldsymbol{\mu}_{\text {rel }}$.

During the months that the magnification is observed, the relative position of the host and the planet can be evolving due 

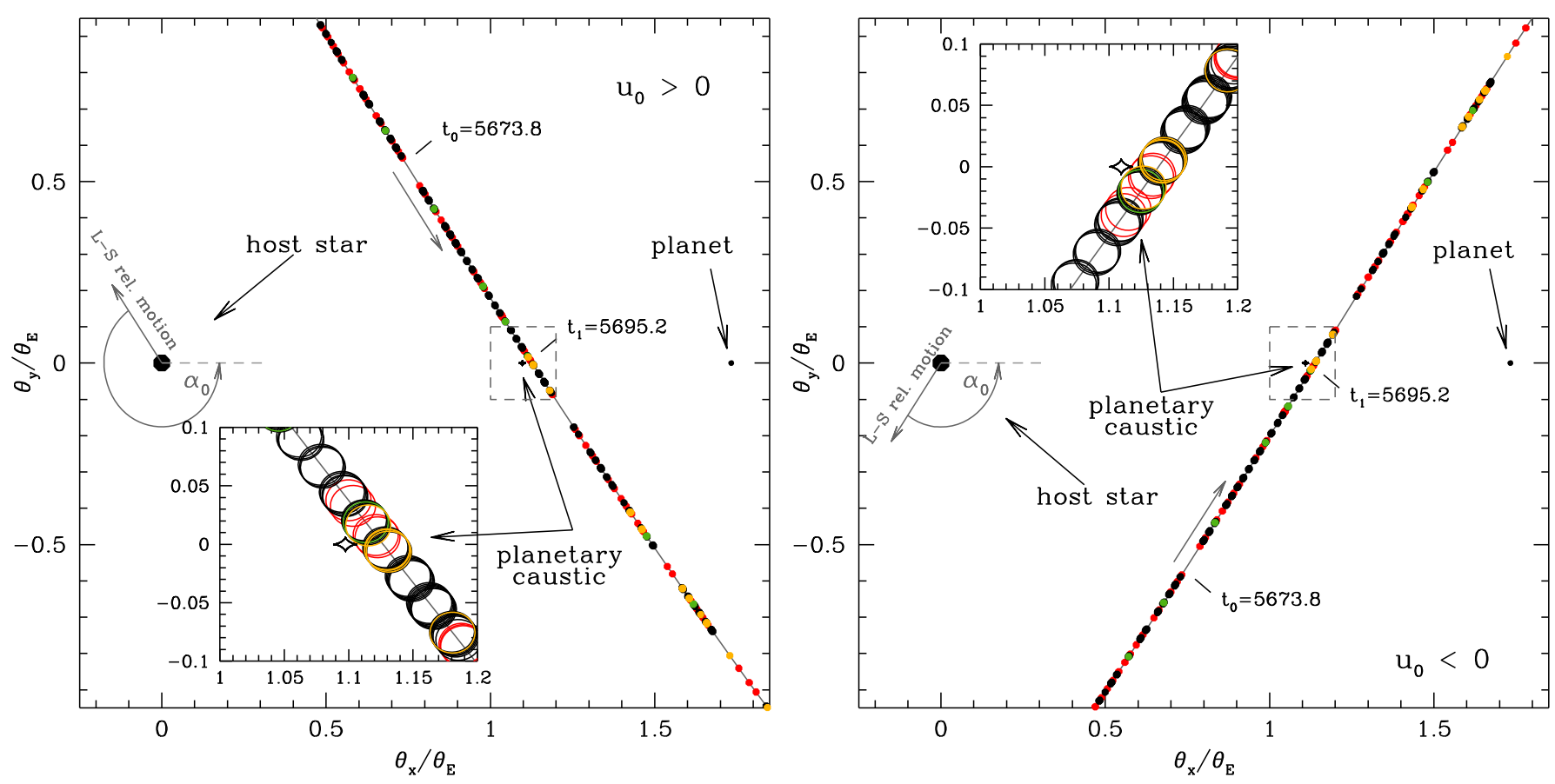

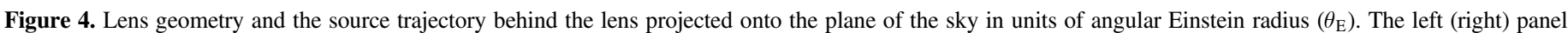

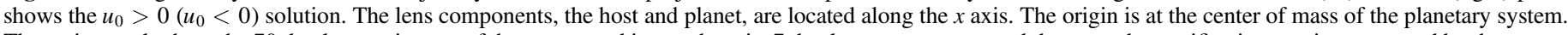

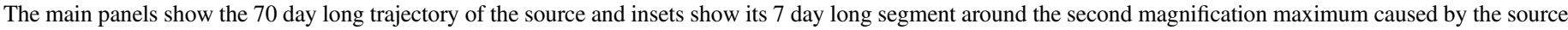

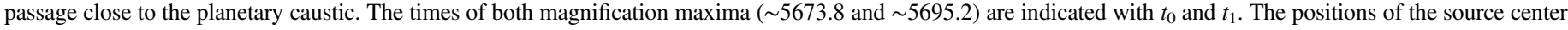

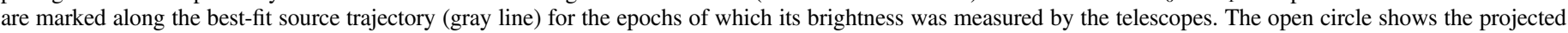

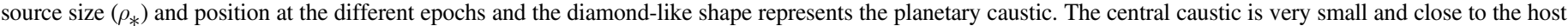
star, and hence is not visible in the plots. The colors follow the conventions of Figure 1.

to their orbital motion. We describe this effect with two constant rates of change: $d s / d t$ and $d \alpha / d t$, for the separation and the projected angle of the planet-host axis, respectively. We choose the center of mass of the lensing system as a reference point for $t_{0}$ and $u_{0}$ and for the center of the coordinates system on the plots.

The observer located on Earth is always experiencing acceleration from the Sun. Thus, for a few months-long microlensing event, even with the absence of the observable acceleration of the source star or of that originating inside of the lensing system, the evolution of the lens-source projected position (as seen by the Earth observer) might not be wellapproximated by the rectilinear motion. Hence, we fix the velocity of the observer frame to the Earth's velocity at the fiducial time $t_{0, \mathrm{par}}=2455678.0$ and use the geocentric parallax formalism (Gould 2004) to describe the observer deviations from this motion. To parametrize the influence of the Earth motion on the event's configuration, we take advantage of the microlens parallax vector $\left(\pi_{\mathrm{E}}\right)$, which has the same direction as the lens-source relative motion, while its magnitude is a ratio of the astronomical unit to the radius of the Einstein ring projected from the source onto the Earth's orbit. It is a useful parameter, as it ties the scale of the Earth's orbit to the scale of the Einstein ring. The simple projection gives:

$$
\mathrm{AU} / D_{\mathrm{L}}-\mathrm{AU} / D_{\mathrm{S}}=\theta_{\mathrm{E}} \pi_{\mathrm{E}}
$$

Note that by combining Equations (1) and (3) we have

$$
M_{\mathrm{L}}=\frac{\theta_{\mathrm{E}}}{\kappa \pi_{\mathrm{E}}} \text {. }
$$

Thus, the measurement of $\pi_{\mathrm{E}}=\left|\pi_{\mathrm{E}}\right|$ and the angular scale of the Einstein ring $\left(\theta_{\mathrm{E}}\right)$ immediately yields the mass of the lensing system and, together with the estimation of the source's distance, provides the distance to the lens (Equation (3)).

\subsection{Extended Source Star}

The planetary anomaly is a result of the source star passing close to the planetary caustic (cf. Figure 4). Due to the finite angular size of the source, the observed magnification pattern is smoothed out in time, while different parts of the star's disk are being strongly magnified by the caustic proximity. To quantify this effect, we use the $\rho_{*}$ parameter, which is the radius of the source's disk with respect to the Einstein ring radius or, equivalently, we use the time $t_{*}$, in which the source star passes the distance of its angular radius $\left(\theta_{*}\right)$. We have the following relations:

$$
\begin{gathered}
\rho_{*}=\frac{\theta_{*}}{\theta_{\mathrm{E}}}=\frac{t_{*}}{t_{\mathrm{E}}} \\
\theta_{\mathrm{E}}=\frac{\theta_{*}}{\rho_{*}}=\theta_{*} \frac{t_{\mathrm{E}}}{t_{*}} .
\end{gathered}
$$

The measurement of this effect from the light curve, while knowing the angular radius of the source (see Section 4.1), allows us to measure the angular size of the Einstein ring $\left(\theta_{\mathrm{E}}\right)$.

For the brightness profile of the disk of the source star, we adopt the square-root limb-darkening law and use the coefficients provided by Claret (2000) in his Table 32 for $v_{t}=2$, solar metallicity, $T_{\text {eff }}=4750 \mathrm{~K}$, and $\log g=2.5-$ as we find appropriate for the red clump giant in the Galactic bulge, for which we observe $(V-I)_{0}=1.04$ (see Section 4.1 
for the characterization of the source star):

$$
\begin{gathered}
c_{I \text {-band }}, d_{I \text {-band }}=0.2530,0.4713, \\
c_{\mathrm{MOA}-R}, d_{\mathrm{MOA}-R}=0.3017,0.4443, \\
c_{V \text {-band }}, d_{V \text {-band }}=0.6035,0.2386 .
\end{gathered}
$$

Coefficients for non-standard MOA- $R$ filter are calculated as a linear combination of $R$-band and $I$-band with $30 \%$ and $70 \%$ weights.

\subsection{Basic Model Parameters from the Light Curve}

Since the coverage of the light curve is very dense and the shape of the anomaly is well-defined, the microlensing nature of the event is clear; furthermore, there have to be at least two bodies in the lensing system. The Einstein timescale of the main brightening is $t_{\mathrm{E}} \approx 33$ days and the impact parameter is $u_{0} \approx 0.9$. The half-duration time of the planetary anomaly is $t_{\text {anom }} \sim 1$ day and its peak is $\delta t \sim 21$ days after the peak of the main feature. If the source star radius is small with respect to the Einstein ring radius, then the Einstein timescale of the planet alone could be approximated by $t_{\text {E,planet }} \sim t_{\text {anom }}$ and, since $t_{\mathrm{E}} \propto \sqrt{M}$, we could guess from these values that the planet-host mass ratio would be

$$
q=M_{\text {planet }} / M_{\text {host }} \approx\left(t_{\text {E,planet }} / t_{\mathrm{E}}\right)^{2} \sim(1 / 33)^{2} \approx 10^{-3},
$$

otherwise it would be an upper limit. Thus, for a stellar-mass host star, the light curve immediately points to a planetary companion of a Jupiter-Sun mass ratio or smaller.

One can estimate the separation of the planetary caustic from the host star as $u_{c}=\sqrt{t_{\mathrm{eff}}^{2}+\delta t^{2}} / t_{\mathrm{E}}=$ $\sqrt{u_{0}^{2}+\left(\delta t / t_{\mathrm{E}}\right)^{2}} \sim 1.1$, and since the planetary anomaly resembles a major image perturbation, we can estimate the planet-host separation from $s-1 / s=u_{c}$ to be $s \sim 1.7$. Also, the angle of the binary axis with respect to the sourcelens trajectory will be $\pm \arctan \left(t_{\mathrm{eff}} / \delta t\right)= \pm 55^{\circ}$. This leads to the angle of the binary axis with respect to the lens-source relative proper motion of $\alpha_{0} \approx(180 \pm 55)^{\circ} \equiv \pm 125^{\circ}$.

The by-eye estimated values of the model parameters $\left(t_{0}, t_{\mathrm{eff}}, t_{\mathrm{E}}, q, s_{0}, \alpha_{0}\right)$ are $\left(5674.0,30,33,10^{-3}, 1.7, \pm 125\right)$, where times are in days and angle in degrees.

\subsection{Light-curve Modeling}

When starting from the estimated values for microlens model parameters it is straightforward to converge to the satisfactory fit to the observed light curve.

We test for other models, e.g., with minor image perturbation, or with binary source, but do not find appropriate explanation of features in the light curve with them. There is, however, a symmetric solution with $u_{0}<0$ (i.e., with the lens passing the source on its left), which is mathematically indistinguishable, from the $u_{0}>0$ solution, in the case of static binary lens (c.f. Skowron et al. 2011).

We use $\chi^{2}$ as a goodness-of-fit measure and use Markov Chain Monte Carlo (MCMC) method for sampling the parameter space around the solutions and evaluation of the parameter uncertainties. Following Skowron et al. (2015), we calculate microlensing magnifications with hexadecapole and inverse ray shooting algorithms, where we use Skowron \& Gould (2012a) method and code library (described in Skowron \& Gould 2012b) for solving the lens equations.
The best-fit model light curve is plotted as a continuous line in Figure 1, and geometry of the event is presented in Figure 4.

\subsection{Non-negative Blended Light}

The term blended light refers to the additional light measured at the position of the microlensing event that was not magnified during its progress. This may be due to several different factors: it might have come from field crowding, where some unrelated star happened to lie inside the source star's seeing disk; it might have come from the distant companion to the source star or the lens; it might have come from the lens itself; or it might be from any combination of these factors. Therefore, the blended light we measure from the light curve is an upper limit on the brightness of the lens (or any other object in the seeing disk); we will make use of this fact later, in Section 4.3.

On the other hand, the best-fit microlensing model reports negative blending at a level of a few percent i.e., the negative amount of additional light is preferred by the mathematical model of the event. In other words, the minimal $\chi^{2}$ is obtained when the source star is brighter than the observed baseline brightness at the position of the event. Then it is possible to obtain the observed maximal brightness at the event's peak with the lower amplification provided by the microlensing model. At the event's baseline, however, when the amplification of the source star is by definition equal to 1 , some negative light needs to be added to recover the lower level of the observed baseline brightness.

The negative source of light is unphysical, thus we limit our solutions to only those with positive blended light. This lower limit ( $\equiv 0)$ on the blend's flux is, at the same time, a lower limit on the peak magnification, so the upper limit on the impact parameter. In our case it translates to: is $\left|u_{0}\right|<0.94$.

Actually, when dealing with the real scientific data, in some fraction of observed microlensing events a low amount of negative blended flux is expected. We recognize that it is possible for the profile photometry, which is performed on the baseline object, to overestimate the background level in the crowded fields of Galactic bulge. This in turn would underestimate the object's brightness and act as a negative source of light. This effect, however, cannot be significantly larger than the faintest objects measured in the field, which for the OGLEIV is $\sim 21 \mathrm{mag}$, and thus could significantly impact only events with very faint sources. In the case of MOA-2011-BLG-028, the source star is very bright - a red clump giant-and $21 \mathrm{mag}$ corresponds to only $0.5 \%$ of the baseline flux.

We treat the solutions with negative blend flux as being allowed solely by the mathematical description of the event, but not as a physical possibility.

\subsection{The Microlens Parallax}

Best-fit models, including the microlens parallax effect, are located very close to the $\pi_{\mathrm{E}} \equiv 0$ with respect to the uncertainties in $\pi_{\mathrm{E}}$. This tells us that the microlensing parallax effect, i.e., the observer's motion along the Earth's orbit, is not detected in the light curve. Actually, this is not surprising, as most of the lenses are located in the Galactic bulge (cf. Dominik 2006); therefore, the Einstein radius projected from the source onto the Earth's orbit is very large. This makes any shift of the Earth's position along the orbit very hard to notice in the light curve unless there are strong magnification features and they are observed throughout a significant length of time 

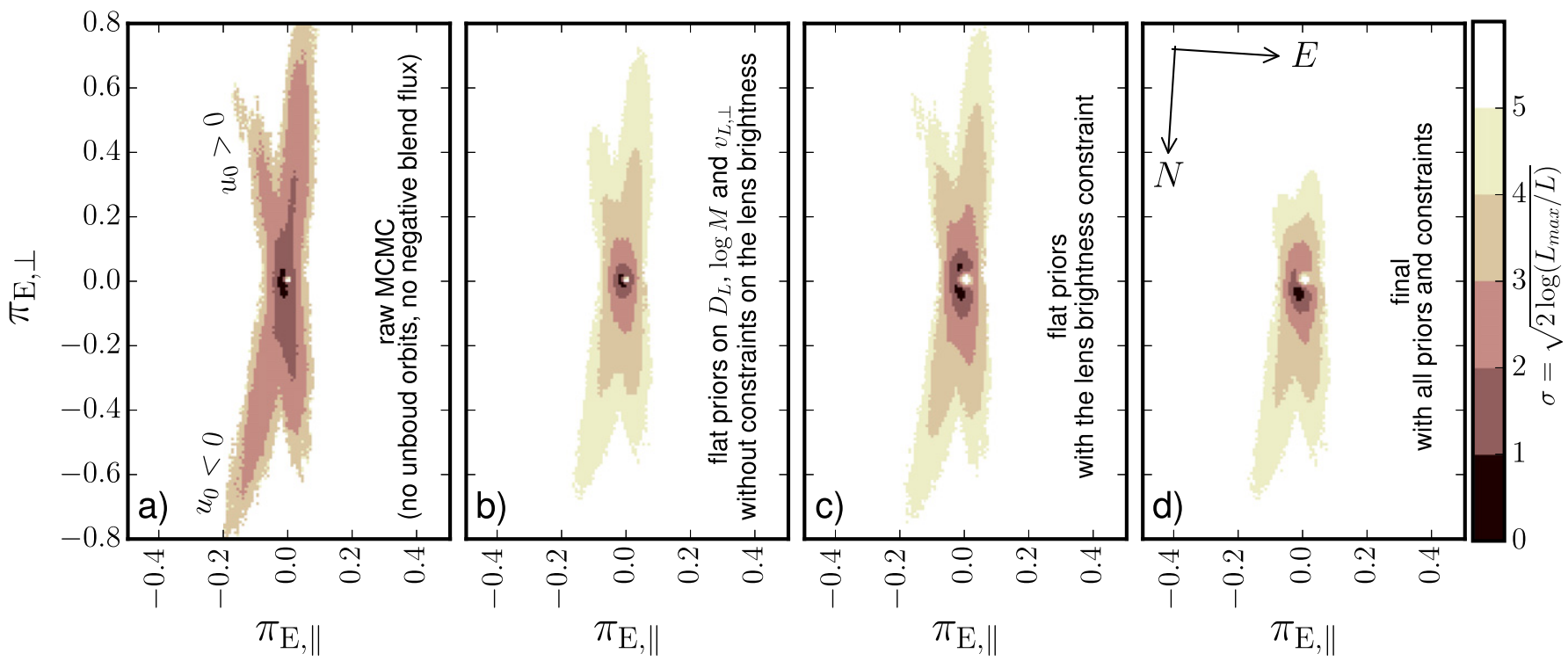

Figure 5. Evolution of the parallax vector posteriors depending on the choice of priors. The $\pi_{\mathrm{E}, \|}$ and $\pi_{\mathrm{E}, \perp}$ are the components parallel and perpendicular to the Earth's acceleration at $t_{0, \text { par }}$ projected onto the plane of the sky. Equatorial north and east directions are indicated in the last panel. Panel (a) shows the projection of all MCMC links for both $u_{0}>0$ and $u_{0}<0$ solutions. Parameter combinations that would yield unbound orbits (projected kinetic energy higher than the projected potential energy as discussed in Section 3.7) or would require negative blended light (Section 3.5) are excluded. Panel (b) shows that the MCMC links are weighted by the lensing probability and the Jacobian of the change from microlensing parametrization to the physical space of distance, mass, and projected velocities (Section 4.4).

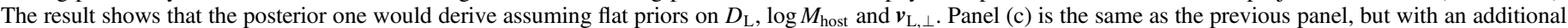
constraint from the mass-luminosity relation for the lens: the distance and the mass of the lens cannot yield the observed $I$-band magnitude that is brighter than the allowed amount of the blended light (Section 4.3). Panel (d) shows all previous constraints together with the priors from the Galactic model, i.e., density profiles of the disk and the bulge, velocities and velocity dispersions of both populations, and the mass function (see Section 4.4 for more details). It is clear that there is no strong tension between the priors and the microlensing light curve solution.

(for instance, in the case of resonant caustic, cf. Skowron et al. 2015).

One can assume that if the microlens parallax is not detected, we do not have any information about it. Therefore, the only recourse is to rely on the statistical expectations on the value of the parallax vector (based on the Galactic models) to estimate the physical parameters of the system (for example Beaulieu et al. 2006; Koshimoto et al. 2014). In reality this should rarely be the case.

While we still need to employ our expectations about the lenses in the Galaxy, we can also use limits on the microlens parallax vector derived from the light curve. In short, not all the values of the parallax vector that we would expect to see from the lens population are consistent with the particular light curve.

Typically these limits are one-dimensional, as it is easier to measure $\pi_{\mathrm{E}, \|}$ (Gould et al. 1994) and they can still rule out noticeable portions of the parameter space, therefore are useful and should be employed (e.g., Gould et al. 2006).

To illustrate this point, the left panel of Figure 5 shows the values of $\pi_{\mathrm{E}}$ that are allowed by the light curve model. Thus, the remaining parameter space is rejected by the light curve.

\subsection{The Orbital Motion of the Lens}

As mentioned earlier, we expect the planet to move in the orbit around its host, and we take this into account by allowing the $\gamma_{\perp} \equiv d \alpha / d t$ and $\gamma_{\|} \equiv(d s / d t) / s_{0}$ to be non-zero. In our modeling, while the best values of $\gamma_{\perp}$ stay around zero for the best models, the $\gamma_{\|}$moves to the negative side with the best-fit value around $-1.5 \mathrm{yr}^{-1}$. While this would indicate a fast orbital motion and near edge-on orbit, the $\chi^{2}$ improvement is only on the order of a few, and zero motion in this direction is still a viable solution and able to explain the shape of the light curve well.

Since it is possible to evaluate mass and the projected separation of the lensing system (see Section 3.1), following Dong et al. (2007) for each trial set of parameters, we calculate the ratio of transverse kinetic to potential energy $\left(\beta=\left|\mathrm{KE}_{\perp} / \mathrm{PE} \mathrm{E}_{\perp}\right|\right)$ and discard all that show $\beta>1$ as obviously unphysical (or unbound) solutions. This reduces the allowed space of the orbital motion parameters to about $\gamma_{\|}=-0.4 \pm 0.4 \mathrm{yr}^{-1}$ and $\gamma_{\perp}=0.0 \pm 0.4 \mathrm{rad} \mathrm{yr}^{-1}$.

For the set of physically bound solutions, the best $\chi^{2}$ improvement with respect to the static binary solution is only about 1.5. Hence, the existence of the orbital motion in the system is not detected. At this point one would argue that it is unnecessary to introduce those additional parameters to the model, but rather the final planetary system parameters should be based on the static binary solution. This might be, however, not always true.

Although for the statistical model, adding additional degrees of freedom and not getting enough improvement in the goodness-of-fit is considered counter-productive, that is only the case if the model is significantly disjointed from the physics. Here we expect some amount of orbital motion in the system.

If we would look at the Jupiter's orbit face-on, the Jupiter would move at a pace of $\sim 0.5 \mathrm{rad} \mathrm{yr}^{-1}$. When seen edge-on with the projected separation of 3 au from the Sun, it would be getting closer in the rate of $2.25 \mathrm{au} \mathrm{yr}^{-1}$, which corresponds to $\gamma_{\|} \sim 0.75 \mathrm{yr}^{-1}$.

It is true that the introduction of the additional parameters to the description of the event increases the uncertainties in the standard parameters, thus limiting the predictive strength of the 
Table 1

Lensing Parameters

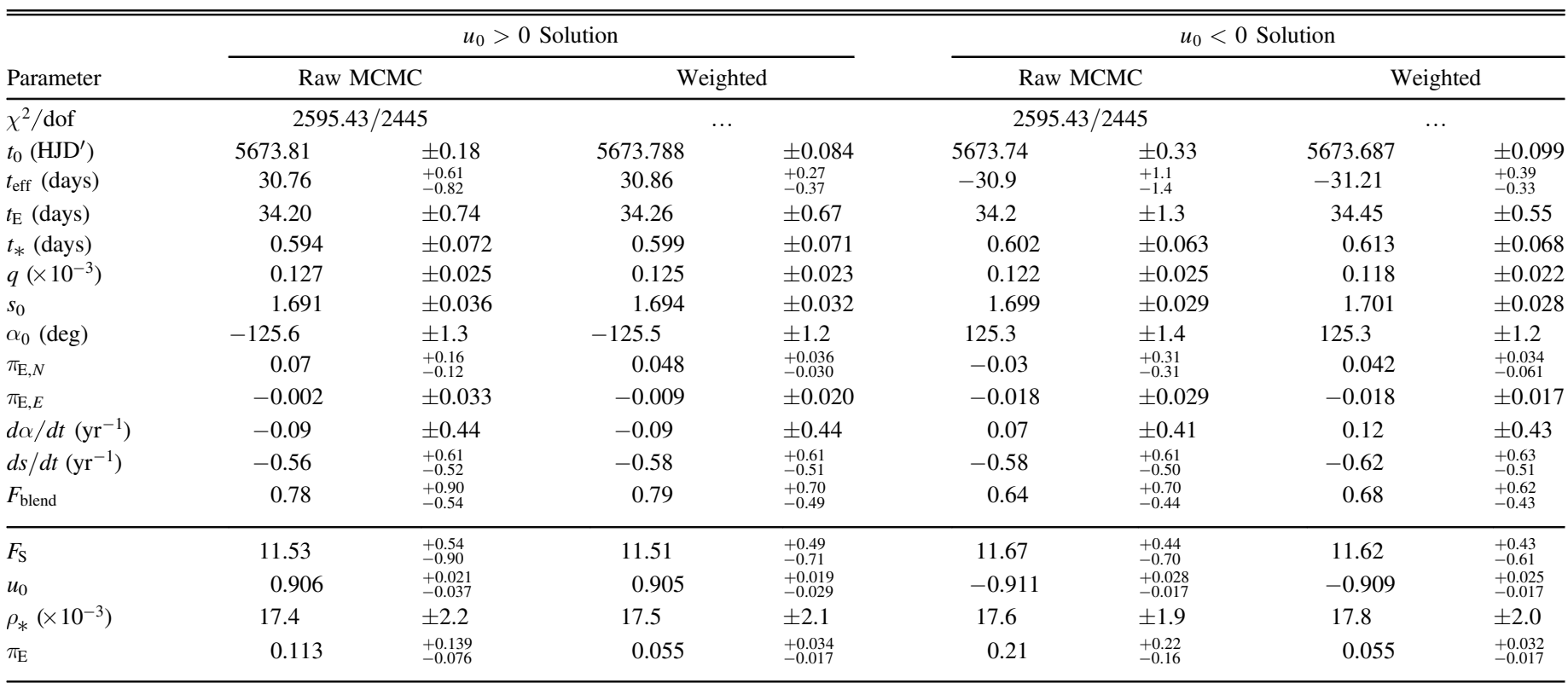

Note. Parameters of the microlensing model. Solutions with both positive $\left(u_{0}>0\right)$ and negative $\left(u_{0}<0\right)$ impact parameters are shown. Raw results from the MCMC modeling are presented with the two constraints on the chain: blended light ("third light") should not be negative (Section 3.5) and kinetic energy projected onto the plane of the sky has to be smaller than the projected potential energy (Section 3.7). The "weighted" parameters are after the inclusion of the geometric weighting, all priors from the Galactic model (see Section 4.4), the source proper motion measurement (Section 4.2), and the limit on the lens brightness (not to exceed the observed blended flux, Section 4.3). After weighting, the $u_{0}>0$ solution holds $68 \%$ of the weight, while $u_{0}<0$ solution holds only $32 \%$. HJD $=$ HJD-2450000. $\alpha_{0}$ and $s_{0}$ denote projected binary axis angle and separation for the epoch $t_{0 \text {,orb }}=5678.0$, respectively. The reference position for the definition of $t_{0}$ and $u_{0}$ is set as the center of mass of the lens system. $u_{0}=t_{\mathrm{eff}} / t_{\mathrm{E}}, \rho_{*}=t_{*} / t_{\mathrm{E}}$ and $I_{\mathrm{b}}=18-2.5 \log F_{\mathrm{b}}$. Geocentric reference frame is set with respect to the Earth velocity at $t_{0, \text { par }}=5678.0$.

model. However, this is actually a very welcome effect as it reflects our ignorance about the system. Avoiding such an increase of the uncertainties might lead to an overestimated confidence in the results.

Here, for the inference of the planetary system physical parameters, we use the full set of fit variables and do not assume static binary system.

\subsection{Resulting Microlensing Parameters}

Table 1 shows our best estimates of the event's microlensing parameters and the uncertainties derived from the MCMC sampling around the two solutions $\left(u_{0}>0\right.$ and $\left.u_{0}<0\right)$. The model consists of the rotating binary lens, the extended source star, and the relative lens-source motion affected by the annual parallax modulation.

Table 2 provides some insights as to how the inclusion of the parallax and orbital motion into the fits influences the results.

It is evident that five of the basic binary-lens parameters $\left(t_{0}\right.$, $\left.t_{\text {eff }}, t_{\mathrm{E}}, s_{0}, \alpha_{0}\right)$ have their uncertainties increased 3-4 times by allowing the parallax to vary within the microlensing fit. The mass ratio $(q)$ and the source-radius crossing time $\left(t_{*}\right)$ are nearly unaffected. While most of the affected parameters are in essence nuisance parameters, the Einstein ring crossing time $\left(t_{\mathrm{E}}\right)$ enters proportionally into the $\theta_{\mathrm{E}}$ estimation (Equation (6)). Note that the uncertainty of the relative lens-source proper motion value is not impacted since $\mu_{\mathrm{geo}}=\theta_{*} / t_{*}$.

The introduction of the orbital motion into the fit does not have a strong effect on the microlensing parameters in the particular case of the event discussed (with the exception of the parameters immediately related to the angle of the binary axis and the host-planet separation). However, it is the case that for certain events, the orbital motion and parallax effects are highly correlated (e.g., Skowron et al. 2011, Figure 5), and thus the orbital motion might influence the results. In that sense, it is profitable to test one's models against such a possibility.

Note also that there is much less unmagnified flux (the blend flux) allowed by the static binary model without the parallax than in all other models. This has implications onto the upper limit of the host star mass (as introduced in Section 4.3) by relying only on the predictions of the simple static model one would reject solutions with higher-mass (brighter) host stars that are perfectly allowed by the more complex models.

\section{CONSTRAINING PHYSICAL PARAMETERS OF THE SYSTEM}

\subsection{Source Star Angular Radius}

From the microlensing fit to the OGLE $I$-band and $V$-band data, we find the observed flux that was magnified in both bands. From that we find the color of the source star to be $\left((V-I)_{S}, I_{S}\right)=(1.829,15.30)$. With the method described by Nataf et al. (2010) we find the centroid of the red clump stars in the 2 arcmin radius around the position of the event $\left((V-I)_{\mathrm{RC}}, I_{\mathrm{RC}}\right)=(1.850,15.379)$. The source star is $0.02 \mathrm{mag}$ bluer and $0.08 \mathrm{mag}$ fainter then the red clump; therefore, it is most likely a K-type red clump star located in the Galactic bulge. The intrinsic color of the red clump centroid is $(V-I)_{\mathrm{RC}, 0}=1.06$ (Bensby et al. 2011). This yields the dereddened color of the source star:

$$
(V-I)_{S, 0}=1.06-0.02=1.04
$$


Table 2

Comparison of the Uncertainties in the Lensing Parameters for Various Models

\begin{tabular}{|c|c|c|c|c|c|c|c|c|}
\hline \multirow{3}{*}{$\begin{array}{l}\text { Parameter } \\
\chi^{2} \\
t_{0}\left(\mathrm{HJD}^{\prime}\right)\end{array}$} & \multicolumn{2}{|c|}{ Static Binary } & \multicolumn{2}{|c|}{ Orbiting Binary } & \multicolumn{2}{|c|}{ Parallax-only } & \multicolumn{2}{|c|}{ Orbiting with Parallax } \\
\hline & \multicolumn{2}{|c|}{2597.2} & \multicolumn{2}{|c|}{2595.3} & \multicolumn{2}{|c|}{2597.2} & \multicolumn{2}{|c|}{2595.3} \\
\hline & 5673.707 & \pm 0.049 & 5673.694 & \pm 0.053 & 5673.82 & $\pm 0.17(\mathrm{p})$ & 5673.81 & \pm 0.18 (p) \\
\hline$t_{\text {eff }}$ (days) & 31.22 & \pm 0.16 & 31.06 & \pm 0.25 & 30.77 & $\pm 0.70(\mathrm{p})$ & 30.70 & $\pm 0.76(\mathrm{p})$ \\
\hline$t_{\mathrm{E}}$ (days) & 33.91 & \pm 0.26 & 34.19 & \pm 0.45 & 34.07 & \pm 0.68 (p) & 34.20 & $\pm 0.74(\mathrm{p})$ \\
\hline$t_{*}$ (days) & 0.585 & \pm 0.046 & 0.592 & \pm 0.068 & 0.576 & \pm 0.054 & 0.594 & \pm 0.072 \\
\hline$q\left(\times 10^{-3}\right)$ & 0.116 & \pm 0.016 & 0.107 & \pm 0.017 & 0.124 & \pm 0.022 & 0.127 & \pm 0.025 \\
\hline$s_{0}$ & 1.6871 & ${ }_{-0.0102}^{+0.0072}$ & 1.705 & ${ }_{-0.045}^{+0.025}(\mathrm{o})$ & 1.676 & ${ }_{-0.025}^{+0.015}(\mathrm{p})$ & 1.693 & ${ }_{-0.037}^{+0.035}(\mathrm{po})$ \\
\hline$\alpha_{0}(\operatorname{deg})$ & -124.90 & \pm 0.16 & -124.22 & ${ }_{-1.54}^{+0.64}(\mathrm{o})$ & -125.23 & $\pm 0.56(\mathrm{p})$ & -125.6 & ${ }_{-1.3}^{+1.4}(\mathrm{po})$ \\
\hline$\pi_{\mathrm{E}, N}$ & \multicolumn{2}{|c|}{$\ldots$} & \multicolumn{2}{|c|}{$\ldots$} & 0.08 & \pm 0.15 & 0.08 & \pm 0.15 \\
\hline$\pi_{\mathrm{E}, E}$ & \multicolumn{2}{|c|}{$\ldots$} & \multicolumn{2}{|c|}{$\ldots$} & -0.001 & \pm 0.032 & -0.002 & \pm 0.033 \\
\hline$d \alpha / d t\left(\mathrm{yr}^{-1}\right)$ & \multicolumn{2}{|c|}{$\ldots$} & 0.31 & ${ }_{-0.53}^{+0.22}$ & \multicolumn{2}{|c|}{$\ldots$} & -0.09 & \pm 0.44 \\
\hline$d s / d t\left(\mathrm{yr}^{-1}\right)$ & \multicolumn{2}{|c|}{$\ldots$} & -0.49 & ${ }_{-0.56}^{+0.79}$ & \multicolumn{2}{|c|}{$\ldots$} & -0.52 & \pm 0.56 \\
\hline$F_{\text {blend }}$ (up. limit) & \multicolumn{2}{|c|}{0.89} & \multicolumn{2}{|c|}{1.57} & \multicolumn{2}{|c|}{2.11} & \multicolumn{2}{|c|}{2.43} \\
\hline
\end{tabular}

Note. Comparison of the uncertainties in the microlensing parameters for four models: the lens as a static binary, the orbiting binary, the static binary with the microlens parallax, and the orbiting binary with the parallax. The inclusion of the orbital motion has a significant effect on the binary separation $\left(s_{0}\right)$ and the angle of the binary axis $\left(\alpha_{0}\right)$ at the reference time $t_{0, \text { orb }}=5678.0$. These values are marked in the table with (o). The introduction of the microlens parallax effect into the fit, while it does not influence the best $\chi^{2}$ value, has a significant impact on the uncertainties in most of the parameters (marked with (p)), with an exception of the sourceradius crossing time $\left(t_{*}\right)$ and mass ration $(q)$. Results show that ignoring the parallax effect in the microlensing fit (even without significant gains in the goodness-offit) leads to an overconfidence in the estimation of the microlensing parameters. Ignoring the orbital motion of the lens in this particular event has a smaller effect, although in events where the parallax is strongly correlated with the orbital motion it might influence the end results. The last row shows $95 \%$ upper limits on the flux of the blend - the simple static binary model allows for much less blended light than more complex models, and thus might exclude more massive (brighter) lenses than are allowed by the other models. Only the parameters for solutions with positive impact parameter $\left(u_{0}>0\right)$ are shown; the other solutions yield analogous results. Parameter definitions are as in Table 1.

and the total reddening toward the Galactic bulge in this small region around the star

$$
\begin{aligned}
E_{(V-I)} & =(V-I)_{\mathrm{RC}}-(V-I)_{\mathrm{RC}, 0} \\
& =1.85-1.06=0.79 .
\end{aligned}
$$

Nataf et al. (2013) provides the mean distance modulus (DM) of the red clump stars toward our line of sight at $\mathrm{DM}=14.52 \pm 0.23$ and the unreddened absolute brightness of the red clump stars, $M_{I, \mathrm{RC}, 0}=-0.11$. This allows us to calculate amount of extinction in the $I$-band toward the field:

$$
\begin{aligned}
A_{I} & =I_{\mathrm{RC}}-\mathrm{DM}-M_{I, \mathrm{RC}, 0} \\
& =15.38-14.521+0.11=0.97,
\end{aligned}
$$

as well as in the $V$-band, $A_{V}=A_{I}+E_{(V-I)}=1.76$. Therefore, the extinction-free brightness of the source star is

$$
\begin{gathered}
V_{0, S}=V_{S}-A_{V}=17.13-1.76=15.37, \\
I_{0, S}=I_{S}-A_{I}=15.30-0.97=14.33 .
\end{gathered}
$$

Following the discussion of the measurement uncertainties of the unreddened color and the extinction-free brightness of the microlensed sources in Skowron et al. (2015; see their Section 4.2 ), we choose the color uncertainty to be 0.06 and brightness uncertainty to be $0.1 \mathrm{mag}$.

With the color-color relation from Bessell \& Brett (1988) and measured $(V-I)_{S, 0}$ of the source star, we find $(V-K)_{S, 0}=2.41 \pm 0.15$. Then the surface brightness calibration provided by Kervella et al. (2004), for the given $V_{S, 0}$ and $(V-K)_{S, 0}$ yields

$$
\theta_{*}=6.08 \pm 0.65 \mu \text { as. }
$$

With the use of Equation (6), this gives the angular Einstein ring radius of

$$
\theta_{\mathrm{E}}=0.337 \pm 0.053 \text { mas. }
$$

\subsection{The Measurement of the Source Proper Motion}

We note that since unmagnified light (blending) in this event is insignificant when compared with the light from the source $\operatorname{star}\left(F_{\text {blend }} / F_{S}=0.07 \pm 0.07\right)$ and since this region of the sky was monitored by the OGLE-III for eight years, it is possible to measure the proper motion of the source star and thus further constrain the microlensing solution.

Analogous to the process described by Poleski et al. (2012), we measure the positions of all stars in the $7^{\prime} \times 7^{\prime}$ region around the event on all science frames of the field BLG196.5 of the OGLE-III survey and derive their proper motions. These motions are relative to some mean frame of motion based on the brighter stars in the field and do not have physical meaning. Under the assumption that the mean proper motion of the Galactic bulge stars toward this direction $((l, b)=(1.7,-3.5))$ is close to being stationary with respect to the Galaxy, we calibrate the motion of all stars in the field to the sample of known Galactic bulge stars. On the colormagnitude diagram we choose 1441 stars in the narrow ellipse around the centroid of the red clump stars region and analyze their motion. Typical uncertainties of the individual proper motions in the sample are $0.4 \mathrm{mas} \mathrm{yr}^{-1}$. The measured mean motion of the red clump giant sample is $(-1.00,0.23) \pm(2.78,2.91){\mathrm{mas} \mathrm{yr}^{-1}}$ in the $(l, b)$ direction. We see that our fiducial reference frame is moving mainly in the direction of Galactic rotation. This is actually expected because this frame is attached to the mix of bright bulge and disk stars in the field. We also measure the bugle velocity 
Table 3

Priors' Influence on Mass and Distance

\begin{tabular}{|c|c|c|c|c|c|c|}
\hline \multirow{3}{*}{$\frac{\text { Choice of Priors }}{\text { No priors (flat) and no cutoff for lens brightness }}$} & \multirow{2}{*}{\multicolumn{2}{|c|}{$\frac{M_{\text {host }}}{\left(M_{\odot}\right)}$}} & \multirow{2}{*}{\multicolumn{2}{|c|}{$\frac{D_{\mathrm{L}}}{(\mathrm{kpc})}$}} & \multirow{2}{*}{\multicolumn{2}{|c|}{$\frac{v_{\perp, 0, \mathrm{~L}, l}}{\left(\mathrm{~km} \mathrm{~s}^{-1}\right)}$}} \\
\hline & & & & & & \\
\hline & 1.39 & $\begin{array}{l}+0.95 \\
-0.75\end{array}$ & 7.32 & $\begin{array}{l}+0.80 \\
{ }_{-0.92}^{+}\end{array}$ & -48 & ${ }_{-120}^{+130}$ \\
\hline No priors (flat on distance, $\log M_{\text {host }}$ and velocities) & 0.78 & \pm 0.35 & 6.90 & $\begin{array}{l}+0.85 \\
-1.02\end{array}$ & 7 & $\stackrel{+}{-155}^{+93}$ \\
\hline With prior on galactic density $\left(D_{\mathrm{L}}\right)$ & 0.92 & \pm 0.31 & 7.42 & ${ }_{-0.60}^{+0.54}$ & -28 & ${ }_{-140}^{+100}$ \\
\hline With priors on galactic density and velocities in the Galaxy & 0.93 & \pm 0.31 & 7.44 & \pm 0.65 & 30 & ${ }_{-120}^{+48}$ \\
\hline With priors on galactic density, $\log M_{\text {host }}$ and velocities $\left(v_{\perp}\right)$ & 0.75 & $\begin{array}{l}+0.36 \\
-0.30\end{array}$ & 7.38 & $\begin{array}{l}+0.53 \\
-0.62\end{array}$ & 40 & $\begin{array}{l}+44 \\
-119\end{array}$ \\
\hline
\end{tabular}

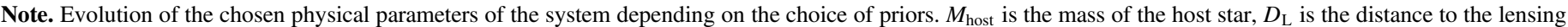

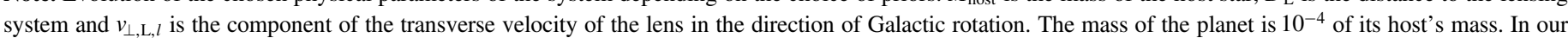

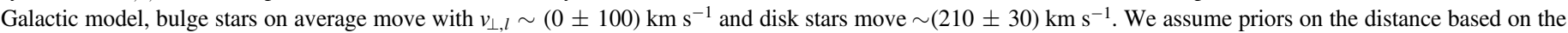

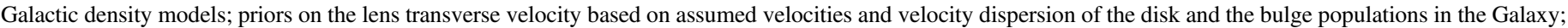

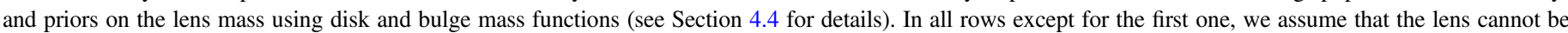
brighter than the amount of the additional/unmagnified light (blend) seen during the microlensing event (Section 4.3).

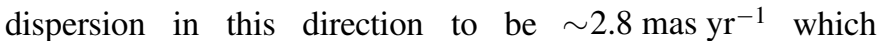
translates to $\sim 106 \mathrm{~km} \mathrm{~s}^{-1}$ and is in agreement with the theoretical models.

The proper motion of the source star in the event MOA2011-BLG-028 is measured to be

$$
\begin{aligned}
\boldsymbol{\mu}_{0, S} & =(-2.79,-1.96)-(-1.00,0.23) \\
& =(-1.79,-2.19) \pm 0.37 \mathrm{mas} \mathrm{yr}^{-1}
\end{aligned}
$$

in the $(l, b)$ direction and is relative to the mean motion of the Galactic bulge stars (which we indicate with an index "0").

\subsection{Upper Limit on the Lens Brightness}

Each trial solution in the MCMC process consists of a set of microlensing parameters (describing the magnification changes) and a blend flux ( $\left.F_{\text {blend }}\right)$. The remaining light from the OGLE-IV baseline is the source star flux $\left(F_{\mathrm{S}}=F_{0}-F_{\text {blend }}\right)$ and is needed to compare theoretical magnification with the observed flux. As we discussed in Section 3.5, we require that $F_{\text {blend }} \geqslant 0$ by rejecting trials with negative blend flux.

In the case where the angular Einstein ring radius is measured (Section 4.1), the solutions with a very low value of $\pi_{\mathrm{E}}$ point to a very high mass of the lens (Equation (4)). In the case of a main-sequence star, this would lead to a very bright lens. There is a limit to which the brightness of such a lens would definitely overcome the blended light seen in the light curve.

For each link in the MCMC, we calculate the distance to the lens (Equation (3)), and from it, its distance modulus $\left(\mathrm{DM}_{\mathrm{L}}\right)$ as well as the mass of the star $\left(M_{\text {host }}\right)$. We use the main-sequence isochrones calibrated by An et al. (2007) and estimate the absolute $I$-band magnitude $\left(M_{I \text {, iso }}\right)$ for the given stellar mass. The lower limit on the brightness of the host star $\left(I_{\text {limit }}\right)$ is set under the assumption that the lens is located behind all the dust, which we measure in front of the source star, and is equal to

$$
I_{\text {limit }}=M_{I, \text { iso }}+\mathrm{DM}_{\mathrm{L}}+A_{I}
$$

where $A_{I}$ is taken from Equation (13). After the conversion to fluxes $(I=18-2.5 \log F)$, we require that

$$
F_{\text {limit }} \leqslant F_{\text {blend }} \text {, }
$$

not to allow the host star to be brighter than the amount of blended light seen in the light curve.
The uncertainty of the mass estimation for each MCMC link reflects the uncertainty in the $\theta_{\mathrm{E}}$. To take this into account, we convolve the less brightness limit together with the $\theta_{\mathrm{E}}$ uncertainty. This process produces a numeric weight with which the particular MCMC link enters into the final considerations.

\subsection{Priors from the Galactic Model}

The measurement of the angular Einstein radius provides a one-dimensional relation between the lens mass and its distance. This degeneracy can be broken by the measurement of the microlens parallax vector length. Unfortunately, in the case discussed here we are unable to detect a clear influence of the Earth's motion onto the light curve. In other words, the best solution is close to the point $\pi_{\mathrm{E}}=0$ (see Table 1 and the first panel of Figure 5). We opt to estimate the physical parameters of the system by employing priors from the Galactic model and, as discussed above, additional information and limits.

We use Jacobian from Batista et al. (2011, Equation (18)) to move the parametrization from the microlensing variables to the physical parameters, the ones in which we have expressed priors. This formula also corrects for the geometric effects of viewing angle and introduces weighting for the lensing probability (which is proportional to the size of the Einstein ring and lens velocity).

For the mass density model of the Galactic bulge, we employ the E3 model by Cao et al. (2013) fitted in the region $|l|<4^{\circ}$, $|b|<4^{\circ}$ to the red clump giants count, the mean distance moduli, and the dispersions measured by Nataf et al. (2013) from the OGLE-III data. We use the Galactic disk mass density model from Han \& Gould (2003) which is based on the $\operatorname{sech}^{2}$ model from Zheng et al. (2001). We also use mass functions described in Appendix B2 of Dominik (2006).

The velocity of the Galactic bulge stars is assumed to be on average zero $\left(v_{l}, v_{b}\right)=(0,0) \mathrm{km} \mathrm{s}^{-1}$ (with respect to the Galaxy) with the dispersion of $100 \mathrm{~km} \mathrm{~s}^{-1}$ in both directions (i.e., the direction of the Galactic rotation and toward the Galactic north). Disk rotation velocity is taken as $220 \mathrm{~km} \mathrm{~s}^{-1}$ with the dispersions of 30 and $20 \mathrm{~km} \mathrm{~s}^{-1}$ in $(l, b)$ directions, respectively. We also consider an asymmetric drift of 10 $\mathrm{km} \mathrm{s}^{-1}$ to account for the fact that the disk stars, on average, rotate a little slower than the Galactic disk gas.

Table 3 presents the influence the different weighing and limits have on the expected distance, mass, and velocity of the 
Table 4

Planetary System Parameters

\begin{tabular}{ll}
\hline \hline Quantity & Final Estimates \\
\hline$M_{\text {planet }}\left(M_{\oplus}\right)$ & $30_{-12}^{+16}$ \\
$M_{\text {host }}\left(M_{\odot}\right)$ & $0.75_{-0.30}^{+0.35}$ \\
$D_{\mathrm{L}}(\mathrm{kpc})$ & $7.38_{-0.62}^{+0.52}$ \\
$a_{\perp}(\mathrm{au})$ & $4.14 \pm 0.64$ \\
$\theta_{\mathrm{E}}(\mathrm{mas})$ & $0.337 \pm 0.053$ \\
$\mu_{\mathrm{geo}}\left(\mathrm{mas} \mathrm{yr}^{-1}\right)$ & $3.59 \pm 0.58$ \\
$\mu_{0, \mathrm{~L}, l}\left(\mathrm{mas} \mathrm{yr}^{-1}\right)$ & $0.68_{-3.24}^{+0.98}$ \\
$\mu_{0, \mathrm{~L}, b}\left(\mathrm{mas} \mathrm{yr}^{-1}\right)$ & $-0.1_{-1.3}^{+1.0}$ \\
$\mu_{0, \mathrm{~S}, l}\left(\mathrm{mas} \mathrm{yr}^{-1}\right)$ & $-1.79 \pm 0.37$ \\
$\mu_{0, \mathrm{~S}, b}\left(\mathrm{mas} \mathrm{yr}^{-1}\right)$ & $2.19 \pm 0.37$ \\
\hline
\end{tabular}

Note. Physical parameters of the lensing system: the mass of the planet in Earth masses $\left(M_{\text {planet }}\right)$, the mass of the host star $\left(M_{\text {host }}\right)$, the distance to the lensing system $\left(D_{\mathrm{L}}\right)$, the projected star-planet separation $\left(a_{\perp}\right)$, the angular Einstein radius $\left(\theta_{\mathrm{E}}\right)$, the relative lens-source proper motion in the geocentric reference frame $\left(\mu_{\text {geo }}\right)$ for time $t_{0, \mathrm{par}}$, the heliocentric len's and source's $\left(\mu_{0, \mathrm{~L}}, \mu_{0, \mathrm{~S}}\right)$ of proper motions with respect to the bulk motion of the Galactic bulge stars. The presented values take into account volume effects as well as priors from the Galactic model: stellar density, velocities, and the mass function (Section 4.4). Since the source's proper motion was measured astrometrically (Section 4.2), it is possible to estimate the lens proper motion. We provide derived values together with standard deviations or $68 \%$ confidence limits where appropriate.

lensing system. Figure 5 shows this in terms of the microlens parallax vector. We see that the lens is most likely located in the Galactic bulge and is a moderately massive star. Also, there is no strong tension between the final parameters of the system and the raw light curve preference.

\section{RESULTS AND DISCUSSION}

Based on the light curve analysis, source star proper motion and priors coming from the expected lens population, we are able to provide assessment of the physical parameters of the planetary system. These are presented in Table 4 . The planet is a Neptune-class planet on the orbit of $\sim 0.75 M_{\odot}$ Galactic bulge star located approximately $7.4 \mathrm{kpc}$ in the direction of $(l, b)=(1.7,-3.5)$. At the time of the lensing event the projected host-planet separation was $4.2 \pm 0.6 \mathrm{au}$.

The source star moves 2.8 mas $_{\mathrm{yr}^{-1}}$ with respect to the mean proper motion of the bulge stars in the field (see Section 4.2) and the relative lens-source proper motion measured from the light curve is 3.6 mas $\mathrm{yr}^{-1}$. Since the majority of the bulge stars

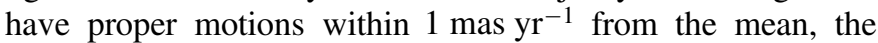
above values are highly compatible with the lens being a typical member of the bulge population. On the other hand, the

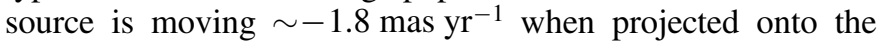
direction of disk rotation, so for the typical disk lens we would expect the relative proper motion of the microlensing event to

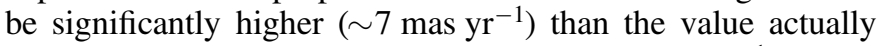
measured from the light curve $\left(\theta_{*} / t_{*}=3.6 \mathrm{mas} \mathrm{yr}^{-1}\right)$.

The most likely location of the source star is slightly behind the mean distance to the bulge (which is $8 \mathrm{kpc}$ in this field, Nataf et al. 2013) at $8.61 \pm 0.64 \mathrm{kpc}$. This is because the lens and the source star are drawn from the same bulge density profile and at the same time the lens must be in front of the source. Also, the probability of lensing by the star in short distance from the source is low.

\subsection{Follow-up Observations}

The extinction in $K_{s}$-band in the discussed direction is estimated by Gonzalez et al. (2012) to be $A_{K s}=0.16$. We take the main-sequence isochrones calibrated by An et al. (2007) to find the absolute $K_{s}$-band magnitude $\left(M_{K_{s} \text {,iso }}\right)$ for the given mass of the host star. This, with the estimation of the distance to the lens, gives the observed magnitude: $K_{s}=M_{K_{s} \text {,iso }}+\mathrm{DM}_{\mathrm{L}}+A_{K s}$. This way we find that the most likely value of the host star's observed brightness is $K_{s}=19.0 \pm 1.4$.

In 2011 April the lens and the source were in near-perfect alignment ( $\leqslant 0.3$ mas). In 10 years from now, the separation of the planetary system from the source giant will be 50 mas-in theory, easy to separate using the ground based AO system or the Space Telescope. Unfortunately, the observed brightness of the source star-a red clump giant toward the Baade Window -is approximately $K_{s}=13$, hundreds of times brighter than the expected brightness of the host star of the planetary system. This makes any follow-up observations very challenging.

As an example, Pietrukowicz et al. (2012) measured the displaced position of the lens with $K_{s}=20.6$ from the source star with $K_{s}=17.4$ at the separation of 125 mas with 37 minutes integration at VLT NACO $(20 \times 110 \mathrm{~s})$. Therefore, the planetary system host (MOA-2011-BLG-028La), if sufficiently separated from the source star, can be detected from the ground. The expected contrast of 6 mag between the lens and the source is much bigger than $3.2 \mathrm{mag}$ in the case studied by Pietrukowicz et al. (2012), requiring a longer wait time before the follow-up observation could be performed or requiring the use of a space-based facility.

However, if the host star is seen in the future, the measurement of its brightness could narrow the mass and distance estimation for this planetary system (e.g., Bennett et al. 2010; Janczak et al. 2010; Fukui et al. 2015). Also, the actual measurement of the lens-source separation and hence, the proper motion, can serve as a useful cross-check for the evaluated here angular size of the Einstein radius, since $\theta_{\mathrm{E}}=\mu t_{\mathrm{E}}$ (see also Section 4.4 of Gaudi 2012).

\section{CONCLUSIONS}

The microlensing event MOA-2011-BLG-028 is a low magnification that peaked in 2011 April in the direction of the Galactic bulge. The source star is a red clump giant, while the lens is most likely a main-sequence star also in the Galactic bulge.

The dense observational coverage of the MOA-2011-BLG028 microlensing event allowed us to confirm the existence of a planetary companion to the main lensing body. The mass ratio is accurately measured to be $(1.2 \pm 0.2) \times 10^{-4}$, indicating a Neptune-class planet.

The finite source effects seen in the light curve allowed us to measure the relative lens-source proper motion. The light curve does not allow for the microlens parallax measurement; however, some limits on this vector value exist.

The low amount of blended light in the light curve and the decade-long OGLE monitoring of the field allowed us to measure the source star proper motion. We use these data as an additional argument for the location of the planetary system inside the Galactic bulge.

We derive the expected physical parameters of the planetary system with the aid of the Galactic model density and the 
velocity distributions. We also weigh our results with the mass function of the potential lenses and the expected lensing rate.

Low-mass as well as moderate-mass main-sequence stars are allowed by the fits. While the moderate-mass and high-mass lenses are preferred by the Galactic density arguments, lenses with a higher mass than $\sim 1.3 M_{\odot}$ are rejected, as the light from them would be clearly detected and thus, are incompatible with observations.

We test our predictions based on the simple static binary microlensing model, as well as more complex models including the parallax and the orbital motion. While none of these effects are proven to be detected in the light curve, a priori, we expect they could have influenced it, as both effects surely are present in the physical reality of all Galactic microlensing events. We see that the fits not allowing for the parallax claim 3-4 times smaller uncertainties of the basic microlensing parameters. Also, the static binary model without the parallax more stringently rejects brighter lenses than more complex models. While the current mass and distance estimations of the planetary system have very wide uncertainties, and it is not proven that the inclusion of the orbital motion is important in this event, the Bayesian analysis with the results of the noparallax static binary model predicts the lensing system that is $\sim 30 \%$ lighter and slightly closer than the system predicted using the models that include the parallax effect.

The host is a $(0.8 \pm 0.3) M_{\odot}$ star located $(7.3 \pm 0.7) \mathrm{kpc}$ away from the Sun in the direction of the Galactic bulge, and is hosting a $12-60 M_{\oplus}$ planet on $\sim 3-5$ au orbit.

The considerable distance to the planetary system and projected proximity of the bright giant star makes it a challenging target for the future follow-up observations.

The OGLE project has received funding from the National Science Centre, Poland, grant MAESTRO 2014/14/A/ST9/ 00121 to A.U.. J.S. was partially supported by the Space Exploration Research Fund of The Ohio State University. This research was partly supported by the Polish Ministry of Science and Higher Education (MNiSW) through the program "Iuventus Plus" award No. IP2011 026771. We thank Prof. M. Kubiak for contributing observations to this work as the OGLE Project member. T.S. acknowledges the financial support from the JSPS, JSPS23103002, JSPS24253004, and JSPS26247023. The MOA project is supported by the grants JSPS25103508 and 23340064. Operation of the Danish $1.54 \mathrm{~m}$ telescope at ESOs La Silla observatory was supported by The Danish Council for Independent Research, Natural Sciences, and by the Centre for Star and Planet Formation. The MiNDSTEp monitoring campaign is powered by ARTEMiS (Automated Terrestrial Exoplanet Microlensing Search; Dominik et al. 2008). N.J.R. is a Royal Society of New Zealand Rutherford Discovery Fellow. J.S. wishes to explicitly thank Prof. Andy Gould for his valuable involvement in the initial phases of this project.

\section{REFERENCES}

An, D., Terndrup, D. M., Pinsonneault, M. H., et al. 2007, ApJ, 655, 233

Batista, V., Gould, A., Dieters, S., et al. 2011, A\&A, 529, A102

Beaulieu, J.-P., Bennett, D. P., Fouqué, P., et al. 2006, Natur, 439, 437

Bennett, D. P., \& Rhie, S. H. 1996, ApJ, 472, 660

Bennett, D. P., Rhie, S. H., Nikolaev, S., et al. 2010, ApJ, 713, 837

Bensby, T., Adén, D., Meléndez, J., et al. 2011, A\&A, 533, AA134

Bessell, M. S., \& Brett, J. M. 1988, PASP, 100, 1134

Bond, I. A., Abe, F., Dodd, R. J., et al. 2001, MNRAS, 327, 868

Cao, L., Mao, S., Nataf, D., Rattenbury, N. J., \& Gould, A. 2013, MNRAS, 434, 595

Cassan, A., Kubas, D., Beaulieu, J.-P., et al. 2012, Natur, 481, 167

Claret, A. 2000, A\&A, 363, 1081

Cumming, A., Butler, R. P., Marcy, G. W., et al. 2008, PASP, 120, 531

Dominik, M. 2006, MNRAS, 367, 669

Dominik, M., Horne, K., Allan, A., et al. 2008, AN, 329, 248

Dominik, M., Jørgensen, U. G., Rattenbury, N. J., et al. 2010, AN, 331, 671

Dong, S., Udalski, A., Gould, A., et al. 2007, ApJ, 664, 862

Fukui, A., Gould, A., Sumi, T., et al. 2015, ApJ, 809, 74

Gaudi, B. S. 2012, ARA\&A, 50, 411

Gonzalez, O. A., Rejkuba, M., Zoccali, M., et al. 2012, A\&A, 543, A13

Gould, A. 2000, ApJ, 542, 785

Gould, A. 2004, ApJ, 606, 319

Gould, A., Dong, Subo., Gaudi, B. S., et al. 2010, ApJ, 720, 1073

Gould, A., Miralda-Escude, J., \& Bahcall, J. N. 1994, ApJL, 423, L105

Gould, A., Udalski, A., An, D., et al. 2006, ApJL, 644, L37

Gould, A., Udalski, A., Shin, I.-G., et al. 2014, Sci, 345, 46

Han, C., \& Gould, A. 2003, ApJ, 592, 172

Harpsøe, K. B. W., Jørgensen, U. G., Andersen, M. I., \& Grundahl, F. 2012, A\&A, 542, A23

Ida, S., \& Lin, D. N. C. 2005, ApJ, 626, 1045

Janczak, J., Fukui, A., Dong, S., et al. 2010, ApJ, 711, 731

Johnson, J. A., Aller, K. M., Howard, A. W., \& Crepp, J. R. 2010, PASP, 122,905

Kennedy, G. M., \& Kenyon, S. J. 2008, ApJ, 673, 502

Kervella, P., Thévenin, F., Di Folco, E., \& Ségransan, D. 2004, A\&A, 426, 297

Koshimoto, N., Udalski, A., Sumi, T., et al. 2014, ApJ, 788, 128

Laughlin, G., Bodenheimer, P., \& Adams, F. C. 2004, ApJL, 612, L73

Mao, S., \& Paczyński, B. 1991, ApJL, 374, L37

Nataf, D. M., Gould, A., Fouqué, P., et al. 2013, ApJ, 769, 88

Nataf, D. M., Udalski, A., Gould, A., Fouqué, P., \& Stanek, K. Z. 2010, ApJL, 721, L28

Paczyński, B. 1986, ApJ, 304, 1

Pietrukowicz, P., Minniti, D., Jetzer, P., Alonso-Garcia, J., \& Udalski, A. 2012, ApJL, 744, L18

Poleski, R., Skowron, J., Udalski, A., et al. 2014a, ApJ, 795, 42

Poleski, R., Soszyński, I., Udalski, A., et al. 2012, AcA, 62, 1

Poleski, R., Udalski, A., Dong, S., et al. 2014b, ApJ, 782, 47

Skottfelt, J., Bramich, D. M., Hundertmark, M., et al. 2015, A\&A, 574, A54

Skowron, J., \& Gould, A. 2012a, General complex polynomial root solver, Astrophysics Source Code Library, ascl:1212.005

Skowron, J., \& Gould, A. 2012b, arXiv:1203.1034

Skowron, J., Shin, I.-G., Udalski, A., et al. 2015, ApJ, 804, 33

Skowron, J., Udalski, A., Gould, A., et al. 2011, ApJ, 738, 87

Sumi, T., Bennett, D. P., Bond, I. A., et al. 2010, ApJ, 710, 1641

Szymański, M. K., Udalski, A., Soszyński, I., et al. 2011, AcA, 61, 83

Udalski, A. 2003, AcA, 53, 291

Udalski, A., Jung, Y. K., Han, C., et al. 2015a, ApJ, 812, 47

Udalski, A., Szymański, M. K., Soszyński, I., \& Poleski, R. 2008, AcA, 58,69

Udalski, A., Szymański, M. K., \& Szymański, G. 2015b, AcA, 65, 1

Woźniak, P. R. 2000, AcA, 50, 421

Zheng, Z., Flynn, C., Gould, A., Bahcall, J. N., \& Salim, S. 2001, ApJ, 555,393 